\title{
Nivel de Consumo de bienes y servicios de índole cultural. Clasificación a través de características socioeconómicas y demográficas de la población paraguaya
}

\section{Level of consumption of goods and services of a cultural nature. Classification through socioeconomic and demographic characteristics of the Paraguayan population}

\author{
Olmedo Barchello, S. ${ }^{1}$ \\ ${ }^{1}$ Dirección de Investigación, Facultad de Ciencias Económicas de la Universidad \\ Nacional de Asunción. Paraguay
}

\section{RESUMEN}

El artículo presenta un análisis descriptivo cuantitativo del nivel de consumo de bienes y servicios de índole cultural de la población paraguaya, los cuales se clasifican en bajo, medio y alto, determinados por el conjunto de bienes y servicios al cual acceden y participan, caracterizándolos de acuerdo a variables socioeconómicas y demográficas. La base de datos utilizada corresponde al módulo cultural introducido en la Encuesta Permanente de Hogares del año 2011, realizado por la Dirección General de Estadísticas, Encuestas y Censos de la República del Paraguay por única vez. Los resultados indicaron que la población paraguaya en su conjunto posee un bajo consumo de bienes y servicios de índole cultural, los cuales se ven reflejados en el sexo, los grupos de edades, las distintas categorías de ingresos, los años de estudios y sobre todo el lugar de residencia. No obstante, los resultados ponen en evidencia la necesidad de una mayor atención a aspectos relacionados a las políticas culturales, inversión, fomento y priorización de programas de desarrollo hacía dichos sectores.

Palabras Clave: Economía; cultura; población; consumo; industrias.

\section{ABSTRACT}

This article presents a descriptive and quantitative analysis of the level of consumption of cultural nature goods and services of the Paraguayan population, which are classified into low, medium and high, determined by the set of goods and services to which it accesses and participates, characterizing them according to socioeconomic and demographic variables. The database corresponds to the cultural module introduced in the Permanent Household Survey of 2011, carried out by the General Directorate of Statistics, Surveys and Censuses of the Republic of Paraguay for once. The study results indicate that the Paraguayan population as a whole has a low cultural consumption, which are reflected in the sex, the age groups, the different categories of income, the years of studies and especially the place of residence. However, the results highlight the need for greater attention to aspects related to cultural policies, investment, promotion and prioritization of development programs towards these sectors.

Keywords: Economy; cultura; population; consum; industry.

*Autor Correspondiente: Selva Olmedo-Barchello. Dirección de Investigación, Facultad de Ciencias Económicas de la Universidad Nacional de Asunción. Paraguay

E-mail: selvaob@eco.una.py

Fecha de recepción: mayo 2017; Fecha de aceptación: julio 2017 


\section{INTRODUCCIÓN}

Los fundamentos teóricos de la economía clásica han demostrado que el consumo de bienes y servicios está sujeto a las leyes de la demanda y a las fuerzas que la subyacen como ser: el precio, los bienes sustitutos o complementarios, además de los gustos y las expectativas de los consumidores (Mankiw, 2002).

Sin embargo, al tratarse del consumo de bienes de índole cultural estos se basan en aspectos que van mucho más allá de lo monetario y son explicados dentro del estudio de la antropología, la sociología y la psicología. En este sentido, García Canclini (2006, p. 42) define a los productos culturales; "como aquellos que poseen valores de uso y de cambio, contribuyendo a la reproducción de la sociedad y a veces a la expansión del capital, cuyos valores simbólicos prevalecen sobre los utilitarios y mercantiles".

Los bienes y servicios culturales, producto de las industrias culturales se reflejan en las artes, las ilustraciones, los desempeños musicales, la literatura, las películas, incluso los programas de televisión, los videojuegos y el turismo cultural (United Nations Conferenceon Trade and Development [UNCTAD], 2008).

El término industrias culturales fue acuñado por Max Horkheimer y Theodor Adorno en 1947 como una crítica radical del entretenimiento de masas, si bien, dicho concepto correspondía a una época donde cultura e industria eran considerados opuestos, actualmente la Organización de las Naciones Unidas para la Educación, la Ciencia y la Cultura (UNESCO) la define como aquellas que "combinan la creación, producción y comercialización de contenidos que son abstractos y de índole cultural (UNCTAD, 2010).

Por lo tanto, las características esenciales de dichos bienes se resumen en los siguientes puntos: su producción requirió un aporte de la creatividad humana, funcionan como vehículo transmisor de mensajes simbólicos y poseen cierto grado de propiedad intelectual.

Bourdieu (2010), clasificó a esta nueva área de estudio como la economía de los bienes culturales, la cual se encarga del estudio de como la sociedad se apropia de los bienes y servicios de índole cultural.

Ahora bien, medir la cultura ha sido siempre un tema de discusión, generalmente por la falta de datos estadísticos, subsanado esta deficiencia a través de diversos modelos de medición, dependiendo por sobre todo de la estructura económica de los países que impulsan las actividades culturales y creativas.

En este sentido, la literatura existente ha prestado especial atención a los estudios de participación cultural de acuerdo a Ateca Amestoy (2009). Es así que las estadísticas de participación han sido un instrumento utilizado con el objeto de conocer el nivel de demanda de dichos bienes con respecto a su oferta, en la mayoría de los casos obteniéndose sólo mediciones y resultados con respecto a su consumo, como ser: el nivel y los motivos de participación, además de los datos socioeconómicos de la población (Aguado, 2010).

En el presente artículo se pretende clasificar el nivel de consumo de bienes y servicios culturales de la población paraguaya de acuerdo a sus características socioeconómicas como; género, edad, años de estudios, nivel de ingresos del núcleo familiar y lugar de residencia. Estos indicadores se analizaron teniendo en cuenta la participación y acceso de los consumidores culturales denominados pasivos de acuerdo a lo sintetizado por Aguado (2010) basándose en el trabajo de O'Hagan en 1996, por lo tanto correspondería a aquellos que asisten a espectáculos en vivo, visita a sitios históricos, consulta a bibliotecas, consumo 
de bienes culturales a través de los medios de comunicación y otros soportes, como; radio, televisión, cine, periódicos, revistas, libros e internet entre otros.

Un estudio exhaustivo sobre demanda cultural, teniendo en cuenta su nivel de gastos, no podría ser factible en esta instancia, por la falta de datos sobre precios y limitaciones de la encuesta utilizada. Sin embargo, cabe destacar que son prácticamente nulos los trabajos que han analizado empíricamente el sector de la economía de la cultural desde una perspectiva de consumo en el Paraguay.

\section{El consumo de bienes y servicios culturales}

Estudiar al consumo cultural se refiere más a un análisis de cómo el individuo se relaciona con la producción simbólica, entendiendo al consumo cultural como "la apropiación de bienes y servicios simbólicos y creativos"(Carlos Catalán, 2005, p. 16, citado en Consejo Nacional de la Cultura y las Artes de Chile, 2011).

En este sentido, Bourdieu (1988) menciona que en el consumo de dichos bienes y servicios la economía de mercado desempeña un papel fundamental al coordinar los esfuerzos de los agentes económicos, cuyas características se ven vinculadas al poder adquisitivo, instrucción, formación o grupo social.

Para Stigler y Becker (1977) la acumulación de experiencias y la inversión en formación son los postulados de su modelo teórico sobre la elección del consumidor, ejemplificado a través del consumo musical, dado por el tiempo asignado a la música y al capital humano musical, este último, producido por la acumulación pasada de apreciación musical y la inversión en educación musical (Aguado y Palma, 2012).

En otras palabras, de acuerdo a Ateca Amestoy (2009), es necesario que exista una dotación inicial de capital humano suficiente para que el consumo de un bien cultural permita producir una experiencia de apreciación cultural, la cual sirva para satisfacer las necesidades del individuo.

Según (Rausell, 2002), el consumo de cultura es un consumo de bienes de experiencia, es decir, las personas no son capaces de determinar antes de consumirlo, si será de su gusto, ni mucho menos la utilidad que les proporcionará, por ello, se buscan mecanismos para reducir riesgos, es decir, recoger información sobre los productos y servicios, revisar sus valoraciones, críticas, opiniones, entre otros.

Este aspecto, refleja el significado de valor cultural (Throsby,2001, p. 33) cuyos orígenes son muy diferentes a los de la esfera económica, en el cual,"el valor subsiste en ciertas propiedades de fenómenos culturales como indicación del mérito o importancia de una obra, un objeto, una experiencia o cualquier otro elemento cultural".

Para Lasuén, García, y Zofío (2005), la historia indica que tanto la composición como las pautas de consumo personal de dichos bienes, se ha ido diversificando y enriqueciendo acorde a los cambios en el nivel de vida de la población, esto como resultado de la variación de las necesidades o de los bienes que los satisfacen, lo cual es difícil de diferenciar y sobre todo de conseguir.

El consumo de estos bienes y servicios, de acuerdo a Bourdieu (2010), están primeramente, relacionadas al nivel de instrucción y segundo al origen social, destacando que esta última ejerce una fuerte influencia. Para Van der Stichele y Laermans (2006), las condiciones estructurales de la sociedad, tales como; ingreso, educación, ocupación, estatus, edad, raza, entre otros, han creado un 
análisis sobre la correlación existente entre el consumo de estos bienes y la estratificación social.

En este sentido, otros autores han considerado a la estratificación social como determinante del consumo cultural estableciendo correlaciones entre frecuencias de consumos de bienes tipificados y las distintas categorías de estratificación de los individuos (Bourdieu y Darbel, 2004; Dimaggio y Useem, 1978).

En el estudio realizado por (Peters, 2010) para el caso de Chile, menciona que ya ha sido demostrado empíricamente la existencia de condicionantes socioeconómicos y estructurales que posibilitan u obstaculizan el consumo cultural de los individuos. A través del mismo se puede conocer la estructura social, teniendo en cuenta la participación y el acceso de la población hacia los productos y servicios culturales, estos a su vez, representan indicadores importantes a las industrias culturales, al realizar un estudio de mercado que indique el tipo de producto de mayor demanda.

Tal como lo menciona Sunkel (2004), el consumo cultural como objeto de investigación estuvo muy vinculado al estudio sobre consumo de medios, el mismo a través de los años resultó en un importante instrumento para el desarrollo de estrategias de mercadotecnia. Por lo tanto, su función consistió en brindar información a los productores para evaluar los resultados de las ofertas existentes.

Los beneficios de un estudio de consumo cultural, indican no solo tener una visión del comportamiento individual sino del país, siendo un instrumento importante a la hora de la toma de decisiones políticas y empresariales. En este sentido, las industrias sobre todo, de índole cultural se han proveído de información con las cuales generar estrategias para mejorar sus ventas, explorar nuevos mercados, productos, además han permitido la regulación, como en el caso de los derechos de autor (Peters, 2010).

Tal como lo mencionan Bernat, Mora, y Zuluaga (2011) basándose en los aspectos metodológicos del trabajo de Muñoz (1995), está última, exploró los hábitos de consumo de cultura en Cali, Colombia, para el que realizó dos tipos de clasificaciones del consumo cultural; el primero en "Externo", donde estudió los hábitos de lectura y la frecuencia del uso de servicios culturales, como; cine, exposiciones y teatro; y el segundo, teniendo en cuenta lo "Doméstico", en el que analizó el consumo habitual de programas de radio y televisión.

Otros autores diferenciaron a las manifestaciones culturales en dos grandes áreas, el de las industrias culturales, incluyendo las industrias del libro, cine, video, fonográfica, además de los medios de comunicación (Cuadrado y Mollá, 2000) y el sector de las artes, constituida por el teatro, la música, la danza, las artes visuales, como las pinturas, escultura y fotografías, además de las bibliotecas, patrimonio y la enseñanza cultural.

Por su parte, la Organización de las Naciones Unidas para la Educación, la Ciencia y la Cultura (UNESCO) en 2014 ha publicado un documento sobre la medición de la cultura, a través de los servicios y los bienes culturales implicados en todas las etapas del ciclo cultural o cadena de valor como la creación, producción, difusión, exhibición, recepción, transmisión, producción y consumo, considerando de este modo a los que conforman los dominios culturales, tales como; el patrimonio cultural y natural, las presentaciones artísticas y celebraciones, las artes visuales y artesanías, los libros y prensa, los medios audiovisuales e interactivos, el diseño y servicios creativos y el patrimonio cultural inmaterial, además de los dominios relacionados con el 
turismo y el deporte, como así también los transversales que incluyen educación y capacitación, archivo y conservación y equipos y materiales de apoyo.

Varios estudios se han realizado en Latinoamérica con respecto al área cultural, enfocados en participación, estudios de hábitos y consumo cultural desde una perspectiva más relacionada a lo antropológico, psicológico, sociológico que a lo económico (Güell, Morales y, Peters, 2010). Por su parte, García Canclini (1993), indicaba que el consumo cultural en América Latina, no era muy estudiado, por lo que se carecía de reflexiones teóricas y datos estadísticos de quienes asistían a los espectáculos, escuchaban música, leían libros, además de otros bienes y servicios culturales.

Entre los estudios que sirvieron de base metodológica para el presente trabajo se destacan los de Dominzain, Rapetti, y Radakovich (2009); Dominzain, Radakovich, Duarte, y Castelli (2014) al realizar estudios en el cual describieron las características socioeconómicas del consumo cultural para Uruguay en el documento denominado "Imaginarios y Consumo Cultural".

Güell, Morales, y Peters (2011) basándose en los datos de la Segunda Encuesta Nacional de Participación y Consumo Cultural de Chile (2011), han analizado el acceso a los bienes y servicios culturales, caracterizando al tipo de consumidor de cultura en; bajo, medio-bajo, medio, alto vía soporte tecnológico y alto general, a través de aspectos socioeconómicos, educativos y etarios de las personas.

\section{Antecedentes de trabajos previos sobre consumo de bienes y servicios culturales en Paraguay}

Entre los primeros documentos se destaca, el denominado "Estudio de exploración de hábitos del consumo cultural" realizado en el año 2008, por el Observatorio Cultural del Centro Cultural de la República el Cabildo, dependiente del Congreso de la Nación Paraguaya. La encuesta utilizada estuvo compuesta por preguntas dirigidas a conocer sobre las actividades culturales con mayor incidencia.

Este estudio, indicó las preferencias de una segmentación bastante joven, residente de un área determinada, en la que la mayoría no poseía ingresos, por lo que las respuestas se mostraron sesgadas y sin cubrir otras actividades culturales como la lectura, la escritura entre otros.

En el 2011, ante la necesidad de tener una mayor información sobre el campo cultural paraguayo, se introdujo el módulo cultural a la Encuesta Permanente de Hogares de dicho año, elaborada por la Dirección General de Estadísticas Encuestas y Censos (DGEEC) y la Secretaría Nacional de Cultura, el cual estuvo compuesto de tres secciones sobre el uso social de la educación y prácticas de literalidad, las prácticas culturales no restringidas formalmente a la competencia en lectura y escritura, las prácticas habitualmente vinculadas a la tradición oral y por último sobre el patrimonio cultural del hogar, siendo el nivel de representatividad del cien por ciento a nivel nacional.

Es así, que con el objetivo de analizar los datos obtenidos en dicha encuesta, en el año 2015, se publicó el documento denominado "El campo cultural paraguayo. Evidencias del módulo de información cultural de la Encuesta Permanente de Hogares 2011", el cual, contenía información sobre prácticas culturales de la población paraguaya, teniendo en cuenta las dimensiones socio demográficas. Este estudio carecía de un análisis de los ingresos de la población encuestada.

Por otro lado, el Informe sobre la Encuesta Latinoamericana de hábitos y prácticas culturales en el año 2014, el cual incluyó a Paraguay, fue elaborada 
por la Organización de Estados Iberoamericanos (OEI) con datos proveídos por la institución denominada Latinobarómetro. La realización de dicho informe surgió de la necesidad de disponer de mayor información relacionada al acceso y participación en actividades culturales en los países de Iberoamérica, además de la valoración sobre la oferta cultural y expectativas futuras. Dicho documento, tuvo una cobertura del cien por ciento de la población del país con alrededor de 1200 casos analizados.

Teniendo en cuenta los estudios previos mencionados, se podía lograr una caracterización de la población paraguaya en cuanto al nivel de acceso y participación por tipo de bien y servicio cultural, teniendo en cuenta aspectos sociales como económicos. No obstante, pensar en el nivel de consumo de dichos bienes, era aún una interrogante que debía de ser respondida y analizada, por lo tanto, a través de una revisión exhaustiva de la literatura y metodologías utilizadas para el análisis de los casos ya referidos para otros países, se desarrolló en este trabajo una metodología que se consideró acorde a los datos con los cuales se contaba para su desarrollo.

\section{MATERIALES Y MÉTODOS}

La investigación realizada fue del tipo descriptivo cuantitativo, cuya base de datos utilizada fue la Encuesta Permanente de Hogares (EPH) del año 2011, específicamente el módulo de información cultural, relacionándolo con el módulo de información demográfica y socioeconómica, obtenidos a través de los microdatos de la Dirección General de Estadísticas, Encuestas y Censos de la República del Paraguay (http://www.dgeec.gov.py/microdatos/index.php). Cabe acotar que la EPH del año 2011 fue la única en incorporar preguntas específicas sobre aspectos culturales de la población paraguaya, no siendo replicado en las encuestas posteriores.

Para su efecto, fueron utilizadas once variables que corresponden a bienes y servicios culturales tales como: "ver la televisión", "escuchar radio", "utilizar internet", "leer el periódico", "leer revistas", "leer libros", "asistir a espectáculos culturales por motivos escolares y profesionales", "asistir a espectáculos culturales por motivos no escolares y profesionales", "visitar sitios históricos y naturales", "consultar bibliotecas", "asistir al cine" que se encontraban en forma de preguntas en el módulo sobre información cultural.

Dichos bienes y servicios fueron seleccionados teniendo en cuenta los principales sectores de las industrias culturales a las que representan; como ser las siguientes: El sector editorial y medios impresos compuesto por: consumo de libros, periódicos, revistas y los servicios de bibliotecas, el sector audiovisual; integran el consumo de cine y televisión, el sector fonográficosonoro, corresponde el consumo de radio, el sector de espectáculos y exposiciones, sean estos danza, teatro, concierto, festividad popular, exposición de dibujo, pintura, o visitas a un museo dentro del país, fuera y por razones escolares o profesionales, el sector patrimonio, considerando al patrimonio tangible como; visita a sitios históricos y naturales y por último el sector de nuevas tecnologías con la utilización de internet.

La periodicidad en el consumo de las mismas no se ha tenido en cuenta, ya que las variables seleccionadas no presentaban en su mayoría uniformidad en la frecuencia y en algunos casos dicha información era nula.

El siguiente paso consistió en clasificar el tipo de consumo en categorías como: Bajo, Medio y Alto, dependiendo de la cantidad de bienes y servicios consumidos, es decir; si el encuestado o encuestada respondió consumir uno o dos bienes y servicios, el tipo de consumo se consideraba "Bajo", en el caso del 
consumo "Medio", se agrupaban aquellos que respondieron haber consumido entre3, 4, 5ó hasta 6 bienes y servicios culturales; y "Alto" sí el consumo fue de $7,8,9,10$ ó hasta 11 bienes y servicios.

El nivel explicativo se da al interpretar, cada uno de los resultados y de manera a vincular el consumo de bienes y servicios culturales a los aspectos socioeconómicos, cuyas variables seleccionadas fueron: sexo (femenino, masculino), edad (distribución por grupos de edad de: 15 a 24 años, 25 a 39 años, 40 a 59 años y a 60 años y más).

El nivel de ingresos del núcleo familiar (menor a 1 salario mínimo, entre 1 y menor a 2 salarios mínimos, entre 2 y menor a 3 salarios mínimos y mayor a 3 salarios mínimos) teniendo en cuenta que el salario mínimo legal vigente en el año 2011 fue de PYG 1.658.232 (Un millón seiscientos cincuenta y ocho mil doscientos treinta y dos guaraníes) y su equivalente en USD 374,40 (Trescientos setenta y cuatro mil dólares americanos con cuarenta centavos), el tipo de cambio correspondiente a dicho año fue en promedio de 1 USD - PYG 4.429, dato obtenido del Banco Central del Paraguay.

Los años de estudios analizados se agruparon en tres grupos de: 1 a 6 años, 7 a 12 años y 13 a 18 años, por otro lado, el lugar de residencia se clasificó en urbano y rural.

El diseño y organización de la base de datos estadísticos fue elaborado por la autora, procesados en el software estadístico-econométrico Stata.

\section{RESULTADOS}

Considerando la cantidad de bienes y servicios de índole cultural que una persona consume de la lista de los once seleccionados, se observó en latabla1, que el $61,3 \%$ consumió entre 1 ó 2 bienes y servicios, correspondiendo el mismo a la categoría "Bajo", el 34,8\% consumió entre 3 a 6 bienes y servicios, es decir, este nivel correspondió al consumo "Medio", y por último el 3,9\% de los encuestados consumió entre 7 a 11 bienes y servicios, siendo los mismos, los de "Alto" consumo cultural.

Tabla 1. Niveles de consumo de bienes y servicios de índole cultural año 2011, en porcentajes

\begin{tabular}{lc} 
Niveles de consumo & $\%$ \\
\hline Bajo & 61,3 \\
Medio & 34,8 \\
Alto & 3,9 \\
\hline Fuente: Elaboración Propia con datos de la Encuesta Permanente de Hogares, año 2011.
\end{tabular}

Una vez agrupados los respectivos niveles de consumo de bienes y servicios de índole cultural del cuadro anterior, se pasó a la fase de caracterización según variables socioeconómicas y demográficas de la población encuestada tal como se observa en la Tabla 2. 
Tabla 2. Niveles de consumo de bienes y servicios de índole cultural por caracterización socioeconómica y demográfica. Año 2011

\begin{tabular}{|c|c|c|c|}
\hline \multirow{2}{*}{$\begin{array}{l}\text { Caracterización } \\
\text { socioeconómica y } \\
\text { demográfica }\end{array}$} & \multicolumn{3}{|c|}{ Niveles de consumo en $\%$} \\
\hline & Bajo & Medio & Alto \\
\hline \multicolumn{4}{|l|}{ Sexo } \\
\hline Femenino & 61,6 & 34,1 & 4,3 \\
\hline Masculino & 60,9 & 35,5 & 3,5 \\
\hline \multicolumn{4}{|l|}{ Edad } \\
\hline 15 a 24 años & 63,1 & 32,6 & 4,3 \\
\hline 25 a 39 años & 51,7 & 42,6 & 5,7 \\
\hline 40 a 59 años & 55,3 & 40,7 & 4,0 \\
\hline 60 y más años & 74,2 & 24,7 & $S / D$ \\
\hline \multicolumn{4}{|c|}{ Nivel de ingresos del núcleo familiar } \\
\hline Menor a 1 SM & 76,9 & 21,8 & 1,3 \\
\hline Entre $1 \mathrm{SM}$ y menor a $2 \mathrm{SM}$ & 61,2 & 36,2 & 2,5 \\
\hline Entre 2 SM y menor a 3 SM & 53,0 & 43,1 & 4,0 \\
\hline Mayor a $3 \mathrm{SM}$ & 54,6 & 38,4 & 6,9 \\
\hline \multicolumn{4}{|l|}{ Años de estudios } \\
\hline 1 a 6 años & 76,4 & 23,3 & $S / D$ \\
\hline 7 a 12 años & 54,2 & 42,2 & 3,6 \\
\hline 13 a 18 años & 40,5 & 46,9 & 12,6 \\
\hline \multicolumn{4}{|l|}{ Lugar de residencia } \\
\hline $\begin{array}{l}\text { Urbana } \\
\text { Rural }\end{array}$ & $\begin{array}{l}55,2 \\
76,0\end{array}$ & $\begin{array}{l}39,6 \\
23,1\end{array}$ & $\begin{array}{l}5,2 \\
S / D\end{array}$ \\
\hline
\end{tabular}

Fuente: Elaboración Propia con datos de la Encuesta Permanente de Hogares, año 2011.

Notas: S/D reflejan la no disponibilidad de datos.

Los resultados reflejan un alto porcentaje en nivel de consumo bajo de bienes y servicios de índole cultural tanto en hombres como mujeres, siendo $61,6 \%$ en el caso de las mujeres y los hombres no alejados con un valor representado por el 60,9\%. En cuanto al consumo medio, fueron los hombres con el 35,5\% quienes aventajaban a las mujeres que llegaban al 34,1\%, el consumo alto poseía predominancia femenina con el $4,3 \%$, siendo el masculino el 3,5\%.

Clasificando por grupos de edades, todos los grupos analizados presentaron altos porcentajes en nivel de consumo bajo, en relación al nivel de consumo medio y alto que disminuyeron. En el caso de la clasificación en nivel de consumo alto, la misma presentó menores porcentajes, reflejando para el último grupo etario, de 60 años y más, la no disponibilidad de datos.

De acuerdo al nivel de ingresos del núcleo familiar los mayores porcentajes se observaron en consumo bajo para todos las agrupaciones, sin embargo, para el último nivel que correspondía a los grupos familiares que percibían más de 
tres salarios mínimos, el porcentaje para consumos medio y alto fueron mayores a las demás categorías.

En cuanto a años de estudios, el porcentaje en consumo bajo resultó mayor para las categorías de 1 a 6 años y 7 a 12 años de estudios, mientras que los que poseían más de 13 años de estudios, el consumo tanto medio como alto resultaron mayores a las demás categorías.

Por último, el lugar de residencia reflejó que las zonas rurales fueron las que poseían mayor porcentaje en cuanto a consumo bajo (76\%), siendo el consumo alto prácticamente inexistente, así también en las zonas urbanas se observó un alto porcentaje de consumo bajo en relación al consumo medio y alto.

\section{DISCUSIÓN Y CONCLUSIONES}

Se pudo constatar que el nivel de consumo de la población paraguaya de bienes y servicios de índole cultural es en general bajo, el consumo medio no supera el $35 \%$ y el consumo alto está representado por una gran minoría.

Desde una perspectiva de género, son las mujeres las poseen un mayor porcentaje en bajo y alto consumo, mientras que el consumo medio resulta mayor en las personas del sexo masculino. En cuanto a la edad, el consumo bajo es mayor en todos los grupos etarios, pero disminuye para los grupos de 25 a 39 años y de 40 a 59 años, incluso los consumos medio y alto reflejan un leve incremento.

Relacionando con el nivel de ingresos del núcleo familiar, el consumo bajo resulta mayor para los que poseen menos de un salario mínimo, en los de consumo medio para los que perciben ingresos entre dos y menos a tres salarios mínimos, mientras que el consumo alto se vuelve mayor para aquellos con más de tres salarios mínimos.

Los años de estudios reflejan la relación existente entre educación y consumo de bienes y servicios de índole cultural, el cual se incrementa en cuanto más años de estudios haya adquirido la persona encuestada. En cuanto al lugar de residencia, son las zonas urbanas las que ofrecen un mayor consumo de dichos bienes y servicios.

Los resultados obtenidos no se ven alejados de las revisiones de trabajos y estudios anteriores analizados, como en el caso de lo expuesto por Bourdieu en 1988 , en el que los agentes económicos analizados y clasificados por nivel de consumo, respondieron a cuestiones vinculadas a su poder adquisitivo, instrucción o nivel de formación, así también por Van der Stichele y Laermans al analizar con aspectos estructurales de la sociedad, además de los trabajos de Peters al demostrar que los condicionantes socioeconómicos y estructurales son los que posibilitan u obstaculizan el consumo cultural de las personas.

El nivel de consumo de bienes y servicios de índole cultural de la población paraguaya, también es reflejo de aspectos relacionados a las políticas culturales, la inversión, el fomento y la priorización de los programas de desarrollo hacía dichos sectores.

Esto también repercute en el sector privado, ya que lo cultural no necesariamente debe ser atendido desde el sector público, son los emprendedores culturales y hoy llamados creativos los que imprimen dinamismo a dicho sector con la innovación tecnológica y nuevos modelos de negocios.

Además pone en evidencia, como está compuesta la población en cuanto a edades, los gustos y preferencias de una población joven difieren de una más madura, los medios de transmisión simbólicos han evolucionado con el correr de 
los tiempos y es en este sentido que las políticas de desarrollo para el sector cultural son las que deberían de acompañar los referidos cambios.

No obstante, es importante avanzar en el proceso de colectar datos y tener estadísticas que permitan a los tomadores de decisiones conocer más sobre este sector, por lo tanto, encuestas que reflejen cuánto dinero destinan los paraguayos al consumo de dichos bienes y servicios o ahondar en la disposición a pagar de cada uno de ellos.

Estos datos podrían considerarse insumos importantes a la hora de plantear y formular políticas públicas, para que a través de los mismos, se permita conocer la estructura económica de la población y la demanda potencial que tendrían las industrias culturales, esto a su vez impulsaría a un sector emergente, diversificaría la estructura económica y dinamizaría la economía en su conjunto.

\section{REFERENCIAS BIBLIOGRÀFICAS}

Aguado, L. (2010). Estadísticas culturales: una mirada desde la Economía de la cultura. Cuadernos de Administración, 23(41), 107-141.

Aguado, L., \& Palma, L. (2012). Una interpretación metodológica sobre la incorporación de los bienes y servicios culturales al análisis económico. Lecturas de Economía, 77, 219-252.

Ateca Amestoy, V. M. (2009). El capital humano como determinante del consumo cultural. Estudios de Economía Aplicada, 27(1), 87-110.

Banco Central del Paraguay. (2011). Cotización referencial histórica de Monedas. Recuperado a partir de https://www.bcp.gov.py/webapps/web/cotizacion/monedas historica

Bernat, L., Mora, J., \& Zuluaga, B. (2011). La elasticidad ingreso del consumo cultural en Cali. Revista de Economía Institucional, 14(27), 165-192.

Bourdieu, P. (1988). La distinción. Criterios y bases sociales del gusto. Taurus.

(2010). El sentido social del gusto. Elementos para una sociología de la cultura (1 a. ed). Buenos Aires, Argentina: Siglo Veintiuno Editores.

Bourdieu, P., \& Darbel, A. (2004). El amor al arte: los museos europeos y su público. Buenos Aires, Argentina: Paidós.

Centro Cultural de la República El Cabildo, \& Asociación Cultural Comuneros. (2008). Observatorio Cultural de la República del Paraguay. Asunción.

Conferencia de las Naciones Unidas sobre Comercio y Desarrollo [UNCTAD]. (2010). Economía Creativa. Una opción factible de desarrollo. Suiza: Naciones Unidas.

Consejo Nacional de la Cultura y las Artes. (2011). Segunda Encuesta Nacional de Participación y Consumo Cultural. Chile: Consejo Nacional de la Cultura y las Artes.

Cuadrado, M., \& Mollá, A. (2000). La relación consumidor-artes: un equilibrio entre satisfacción de necesidades y libertad creativa. Estudios sobre Consumo, 15(53), 23-32.

Dimaggio, P., \& Useem, M. (1978). Social Class and Arts Consumption: The Origins and Consequences of Class Differences in Exposure to the Arts in America. Theory and Society, 5, 141-161.

Dirección General de Estadísticas, Encuestas y Censos. (2016). Microdatos - Encuesta Permanente de Hogares [Oficial]. Recuperado a partir de http://www.dgeec.gov.py/microdatos/index.php

Dominzain, S., Radakovich, R., Duarte, D., \& Castelli, L. (2014). Imaginarios y Consumo Cultural (Tercer Informe Nacional sobre Consumo y Comportamiento Cultural). Uruguay: Ministerio de Educación y Cultura y Universidad de la República.

Dominzain, S., Rapetti, S., \& Radakovich, R. (2009).Imaginarios y Consumo Cultural. Segundo Informe Nacional sobre Consumo y Comportamiento Cultural (No. Segundo). Uruguay: Ministerio de Educación y Cultura.

García Canclini, N. (1993). El consumo cultural y su estudio en México: una propuesta teórica. México: Conaculta.

(2006). El Consumo Cultural: Una propuesta teórica. En El Consumo Cultural en América Latina. Construcción Teórica y líneas de investigación 
(Segunda, pp. 72-95). Colombia: Convenio Andrés Bello. Recuperado a partir de http://books.google.com.py/books?hl=es\&lr=\&id=js9eKdJbyREC\&oi=fnd\&pg=PA72 $\& d q=$ consumo + cultural + garcia +canclini\&ots $=$ XbcJWgkoKi\&sig=OFMvONMIYfizdsp 7 P3G_uuft47M\#v=onepage\&q=consumo\%20cultural\%20garcia\%20canclini\&f $=$ false

Güell, P., Morales, R., \& Peters, T. (2010). Una canasta básica de consumo cultural para América Latina: Operacionalizando el Derecho a la participación en la cultura. Centro de Investigaciones Socioculturales - Universidad Alberto Hurtado.

Güell, P., Morales, R., \& Peters, T. (2011). Tipología de prácticas de consumo cultural en Chile a inicios del siglo XXI: mismas desigualdades, prácticas emergentes, nuevos desafíos. Universum, 2(26), 121-141.

Lasuén, J., García, M., \& Zofío, J. (2005). Cultura y Economía. Madrid: Fundación Autor.

Mankiw, G. (2002). Principios de Economía. Madrid: Mc Graw Hill.

Organización de las Naciones Unidas para la Educación, la Ciencia y la Cultura [UNESCO]. (2014). Cómo medir la participación cultural (Manual del Marco de Estadísticas Culturales de la UNESCO 2009 No. 2). Montreal: Instituto de Estadística de la UNESCO.

Palma, L. A., \& Aguado, L. F. (2010). Economía de la Cultura: Una nueva área de especialización de la Economía. Universidad Externado de Colombia, 12(22), 129165.

Peters, T. (2010). La afinidad electiva entre consumo cultural y percepción sociocultural : el caso de Chile. Signo y Pensamiento, XXIX, 216-235.

Rausell, P. (2002, julio). Los mercados culturales y el desarrollo de la nueva economía. Recuperado 7 de abril de 2015, a partir de http://www.uoc.edu/culturaxxi/esp/articles/rausell0602/rausell0602_imp.html

Rebolledo, P. (2013). Determinantes de la Participación en Actividades Culturales para Chile (Tesis de Grado de Magíster en Economía). Pontificia Universidad Católica de Chile, Santiago, Chile.

Secretaría Nacional de Cultura. (2015). El Campo Cultural pParaguayo. Evidencias del Módulo de Información Cultural de la Encuesta Permanente de Hogares 2011. Asunción - Paraguay.

Stigler, G. J., \& Becker, G. S. (1977). De Gustibus Non Est Disputandum. The American Economic Review, 67(2), 76-90.

Sunkel, G. (2004). El consumo cultural en la investigación en comunicaciones en América Latina. Signo y Pensamiento, XXIII, 8-24.

Throsby, D. (2001). Economía y Cultura (Primera). Madrid: Cambridge University Press.

United Nations Conference on Trade and Development.(2008). Creative Economy. The Challenge of assessing the creative economy: towards informed policy-making. New York, USA. Recuperado a partir de http://unctad.org/en/Docs/ditc20082cer_en.pdf

Van der Stichele, A., \& Laermans, R. (2006). Cultural Participation in Flanders: Testing the Cultural Omnivore Thesis with Population Data. Poetics, 34, 45-64. 